\title{
Management of a 75-Year-Old Lady with Refractory Chronic Myelogenous Leukemia
}

\author{
Philip T. Sobash ${ }^{a} \quad$ Achuta K. Guddati $^{b}$ Vamsi Kota ${ }^{b}$ \\ anternal Medicine, White River Health System, Batesville, AR, USA; b Medical Oncology, \\ Augusta University, Augusta, GA, USA
}

\section{Keywords}

Refractory · Tyrosine kinase inhibitors · Chronic myelogenous leukemia · Complete molecular remission - Major molecular response

\begin{abstract}
Chronic myelogenous leukemia (CML) is a hematopoietic disorder caused by the $B C R / A B L$ gene or Philadelphia chromosome. The first Food and Drug Administration (FDA)-approved tyrosine kinase inhibitor for treatment of CML was imatinib in 2001. Since then, multiple therapies, such as nilotinib, dasatinib, bosutinib, and, more recently, ponatinib, have made their way as viable treatment options for first-line and secondary therapies. Most patients tend to respond to first-line treatment. Although there is a subset of patients who do not achieve complete molecular response with first line, newer options have proven beneficial. As we progress through therapies, there still remain some patients who do not adequately respond to current available therapies. The treatment options and guidelines become more difficult in such situations, not only with respect to cost but also patient quality of life and satisfaction. We discuss a 75-year-old white female with CML, who has had multiple therapies with hematological remission but has never achieved complete molecular remission, currently on bosutinib and tolerating it well.

(C) 2020 The Author(s).

Published by S. Karger AG, Basel
\end{abstract}

\section{Introduction}

Chronic myelogenous leukemia (CML) is a myeloproliferative disorder of hematopoietic cells caused by chromosomal abnormalities, specifically the Philadelphia chromosomet $(9 ; 22)$ [1]. Current Food and Drug Administration (FDA)-approved therapies include the tyrosine kinase inhibitors (TKIs) imatinib, nilotinib, dasatinib, and bosutinib [2]. Even with these ther- 
Sobash et al.: Management of Refractory CML

apies, approximately $20-30 \%$ of patients fail to have a complete cytogenetic response on first-line imatinib [2,3]. Salvage therapy includes second- and third-generation TKIs, but there is a mixed response as they can be more selective depending on a variety of patient factors [2].

Bosutinib was primarily studied and evaluated for patients who had an inadequate response to imatinib [4]. Studies evaluating long-term use of bosutinib have shown $84 \%$ overall survival, with most adverse events happening within the first 2 years of therapy [5]. With third-generation TKIs, such as ponatinib, showing promising responses in patients with extensive prior treatment [6], it is difficult to draw the line between searching for complete cytogenic remission and the risk of continually changing therapies, particularly in elderly individuals. We discuss the treatment of an elderly woman who has had suboptimal responses to many first- and second-line therapies, currently on bosutinib for 5 years with relatively stable results.

\section{Case Presentation}

We present a 75-year-old white female with refractory CML, diagnosed in 2004, who has gone through multiple BCR/ABL inhibitors, namely imatinib, nilotinib, and dasatinib, currently on bosutinib $300 \mathrm{mg}$ daily for 5 years who has achieved hematological remission but has never achieved complete molecular response (CMR).

The patient was found to have elevated white blood cell (WBC) count in June of 2004. Subsequent bone marrow aspirate and biopsy were diagnostic for CML. Therapy was initiated with imatinib and was effective until 2007 when her WBC count started to rise again. She was then started on nilotinib $400 \mathrm{mg}$, to which she responded but again subsequently regressed, with her WBC count going from 13,500 to $40,000 / \mathrm{mL}$. Dasatinib was started, which the patient did not tolerate, and was stopped after 1 month due to cardiac symptoms which the patient described as her heart feeling like it was going to "flop out." At this point in time, she was placed on interferon- $\alpha$, but it was stopped due to side effects.

She was seen by our center in 2012 and had recently been placed back on imatinib 800 $\mathrm{mg}$, which was subsequently lowered to $600 \mathrm{mg}$ a day, as well as hydroxyurea. A bone marrow biopsy done in January of 2012 showed chronic-phase CML. Until then, no mutational analysis had been done to evaluate her drug resistance to TKIs. At this time, she also developed pericardial and pleural infusion, most likely from imatinib, and underwent a pericardial window placement in the same month.

Follow-up with mutational analysis was negative, and it was decided to retry dasatinib, as she had progressed through imatinib and nilotinib. Dasatinib 50 mg daily was started with close monitoring and plans to use pulse steroids and diuresis if fluid buildup should develop. She developed a cough relieved by steroids with no effusions present. In March 2013, she was tolerating dasatinib, and her dose was increased to 50/100 mg every other day. BCR/ABL PCR done at this time was $31.83 \%$. BCR/ABL continued to be stable, but no decline in levels was noted. Bone marrow biopsy in May 2013 was negative for BCR/ABL, but the PCR was still concerning. It was decided to try a course of ponatinib at $15 \mathrm{mg}$ daily. After initiation of therapy, she had a decrease in her platelet counts. She was switched to bosutinib in January 2014 due to ponatinib being taken off the market. After the switch to bosutinib, her BCR/ABL clones once again started to increase. A switch back to dasatinib was offered, but the patient was reluctant, and it was decided to continue on bosutinib.

At this point, it was decided not to monitor her BCR/ABL levels as she was not a transplant candidate. The focus was on hematological remission alone. Since that time, the patient's only major complaint with bosutinib has been some bouts of diarrhea, and even this has mainly 
Sobash et al.: Management of Refractory CML

been when dosage increase was attempted. BCR/ABL levels rose from 44\% in 2015 to $65 \%$ in February of 2016. Kinase domain mutation analysis was negative again. BCR/ABL decreased from 65 to $52.3 \%$ in May 2016. At this time, her bosutinib was increased from 300 to $500 \mathrm{mg}$ every day due to another rise in BCR/ABL. This elevated dose caused an increase in diarrhea, and it was dropped down to $300 \mathrm{mg}$ as it was previously. Since that time in 2016, her BCR/ ABL waxed and waned, but she continued in hematological remission. Currently, she is still on bosutinib $300 \mathrm{mg}$ daily with plans to add interferon- $\alpha$ if disease progression is observed.

\section{Discussion}

Treatment options for those with inadequate responses to frontline TKIs remain a substantial clinical problem in most patients. Although age has been shown to be an independent prognostic indicator, studies have not demonstrated any association of BCR/ABL mutations leading to TKI resistance [6]. In our patient, no BCR/ABL mutations have been shown, leading to further unknowns about her future clinical course. With limited availability of therapeutics after exhausting current options, treatment can become a burden on the provider and the patient.

In the case of our patient, mutational analysis has yielded no results to date. Specific therapies, such as the use of ponatinib for T315I kinase domain mutation [7], have been beneficial for a subset of patients who do not respond to initial first- and second-line therapies. There are other treatments currently in clinical trials which have yielded promising results for a slightly different mechanism of action from other TKIs [8]. Our case presents an interesting view of mutation-negative disease that still has not responded to many treatments. Ponatinib is a potential option that has not yet been explored, although it is against our patient's wishes to keep switching treatment regimens due to being relatively stable on current therapy. Research has been conducted to assess TKI discontinuation [9] but is limited in terms of continuation of current therapy despite lack of remission.

A further consideration is the choice between CMR versus major molecular response. Studies in Japan and France have shown that there is no harm in stopping therapy in patients with a major molecular response, and this may be just as effective, and cheaper, than waiting for a CMR [9]. There is emerging evidence that criteria for consideration of manageable levels of remission could be more lenient to allow for wider clinical judgment. While no studies have been conducted to assess the continuation of therapy despite CMR, we postulate that, when therapeutic exhaustion has occurred, remaining on a tolerable dose and monitoring BCR/ABL PCR levels is a clinically suitable option, especially given patient social factors, such as age and unwillingness to change therapies.

Although CMR is the goal in the treatment of CML, this goal must be assessed in the setting of the patient's preferences and after assessing how the patient responds to the therapeutic regimen. CML patients who have evidence of CMR still have evidence of leukemic disease on PCR even after imatinib discontinuation [10]. So, in this light, it could be argued that patients with CML are never fully cured as we might proclaim; rather, we have merely attached this label to certain patient populations. We surmise evidence from our patient falls in line with what John Goldman stated in his 2006 paper, if a therapy can prevent recurrence and resistance, you can provide an "operational cure" [11].

\section{Statement of Ethics}

The patient had consented to the publication of her clinical history and case details. 
Sobash et al.: Management of Refractory CML

\section{Disclosure Statement}

No conflicts of interest exist for any of the authors.

\section{Funding Sources}

There are no funding sources reported.

\section{Author Contributions}

Philip T. Sobash, MD: literature review; wrote the manuscript, revised the manuscript. Achuta K. Guddati, MD, PhD: literature review; wrote the manuscript, revised the manuscript. Vamsi Kota, MD: conceived the study; literature review; wrote the manuscript, revised the manuscript.

\section{References}

1 Sawyers CL. Chronic myeloid leukemia. N Engl J Med. 1999;340(17):1330-40.

2 Jabbour E, Kantarjian H. Chronic myeloid leukemia: 2016 update on diagnosis, therapy, and monitoring. Am J Hematol. 2016;91(2):252-65.

3 Schiffer CA. BCR-ABL tyrosine kinase inhibitors for chronic myelogenous leukemia. N Engl J Med. 2007; $357(3): 258-65$.

4 Cortes JE, Kantarjian HM, Brümmendorf TH, Kim DW, Turkina AG, Shen ZX, et al. Safety and efficacy of bosutinib (SKI-606) in chronic phase Philadelphia chromosome-positive chronic myeloid leukemia patients with resistance or intolerance to imatinib. Blood. 2011;118(17):4567-76.

5 Gambacorti-Passerini C, Cortes JE, Lipton JH, Kantarjian HM, Kim DW, Schafhausen P, et al. Safety and efficacy of second-line bosutinib for chronic phase chronic myeloid leukemia over a five-year period: final results of a phase I/II study. Haematologica. 2018;103(8):1298-307.

6 Hochhaus A. Educational session: managing chronic myeloid leukemia as a chronic disease. Hematology Am Soc Hematol Educ Program. 2011;2011:128-35.

7 Miller GD, Bruno BJ, Lim CS. Resistant mutations in CML and Ph(+)ALL -- role of ponatinib. Biologics. 2014;8: 243-54.

8 Cortes J, Talpaz M, Smith HP, Snyder DS, Khoury J, Bhalla KN, et al. Phase 1 dose-finding study of rebastinib (DCC-2036) in patients with relapsed chronic myeloid leukemia and acute myeloid leukemia. Haematologica. 2017;102(3):519-28.

9 Saußele S, Richter J, Hochhaus A, Mahon FX. The concept of treatment-free remission in chronic myeloid leukemia. Leukemia. 2016;30(8):1638-47.

10 Ross DM, Branford S, Seymour JF, Schwarer AP, Arthur C, Bartley PA, et al. Patients with chronic myeloid leukemia who maintain a complete molecular response after stopping imatinib treatment have evidence of persistent leukemia by DNA PCR. Leukemia. 2010;24(10):1719-24.

11 Goldman J, Gordon M. Why do chronic myelogenous leukemia stem cells survive allogeneic stem cell transplantation or imatinib: does it really matter? Leuk Lymphoma. 2006;47(1):1-7. 